\title{
Epidermal growth factor receptor expression analysis in chemotherapy-naive patients with advanced non-small-cell lung cancer treated with gefitinib or placebo in combination with platinum-based chemotherapy
}

\author{
Giuseppe Giaccone $\cdot$ Renee B. Iacona \\ Abderrahim Fandi • Mette Janas • Judith S. Ochs • \\ Roy S. Herbst • David H. Johnson
}

Received: 23 June 2008 / Accepted: 12 August 2008 / Published online: 12 September 2008

(c) The Author(s) 2008. This article is published with open access at Springerlink.com

\begin{abstract}
Purpose Two large, randomized, placebo-controlled trials (IRESSA NSCLC Trial Assessing Combination Therapy; INTACT 1 and 2) in non-small-cell lung cancer (NSCLC) failed to show survival benefit for gefitinib (IRESSA) in combination with first-line platinum-based chemotherapy. Epidermal growth factor receptor (EGFR) staining was
\end{abstract}

IRESSA is a trademark of the AstraZeneca group of companies.

PharmDx assay is a trademark of Dako.

\section{G. Giaccone}

Free University Medical Center, Amsterdam, The Netherlands e-mail: giacconeg@mail.nih.gov

R. B. Iacona $(\square) \cdot$ A. Fandi $\cdot$ J. S. Ochs

AstraZeneca Pharmaceuticals, 1800 Concord Pike, C2B-505, Wilmington, DE 19850, USA

e-mail: Renee.Iacona@astrazeneca.com

A. Fandi

Novartis Oncology, Florham Park, NJ, USA

A. Fandi

University of Turin, Turin, Italy

M. Janas

Dako, Glostrup, Denmark

Present Address:

M. Janas

Leo Pharma, Copenhagen, Denmark

R. S. Herbst

University of Texas MD Anderson Cancer Center,

Houston, TX, USA

D. H. Johnson

Vanderbilt University Medical School, Nashville, TN, USA assessed retrospectively in relation to survival response to gefitinib in combination with chemotherapy.

Methods Tumor biopsies obtained prior to start of therapy were assessed by immunohistochemistry for EGFR using the Dako EGFR pharmDx assay ${ }^{\mathrm{TM}}$ (Dako, Denmark). Analyses were stratified by trial and performed independently for patients randomized to placebo and gefitinib as well as for both treatment groups combined. A restricted backwards elimination Cox regression analysis was conducted to identify independent EGFR factors that were statistically significant $(P<0.10)$, and these were also tested for treatment interaction to assess if they served as predictive factors.

Results Analyses found two statistically significant EGFRbased prognostic factors representing growth pattern and percent membrane staining in patients treated with gefitinib $(P=0.0023)$, placebo $(P=0.0128)$, and both combined $(P<0.0001)$. The prognostic effect was independent of other known prognostic factors. There was no predictive effect of either the growth pattern or membrane staining variable.

Conclusions While some previous studies indicate that higher EGFR expression correlates with poor survival, our analyses provide statistically significant evidence that the combination of EGFR expression and growth pattern is a strong prognostic indicator for improved survival within this setting. The effects of membrane staining and growth pattern are still significant when adjusting for mutation.

Keywords EGFR - NSCLC - Gefitinib - Chemotherapy · Tumor biopsies $\cdot$ Immunohistochemistry $\cdot$ Biomarkers

\section{Introduction}

The identification of factors that may predict survival (i.e., prognostic factors) or response to treatment (i.e., 
predictive factors) of non-small-cell lung cancer (NSCLC) is an important goal, as it may, in the future, enable identification of patients with better prognosis, or targeting of treatment to those patients most likely to respond. One such factor worthy of consideration is the epidermal growth factor receptor (EGFR), a member of the erbB family of receptors. EGFR, which is expressed or highly expressed in a variety of solid tumors, has been implicated in the pathogenesis of human malignancies and hence is a rational target for anticancer therapies (Salomon et al. 1995; Woodburn 1999). Expression of EGFR may be demonstrated by means of different techniques; for example, immunoblotting and immunohistochemistry (IHC). Even within one technique, differences in primary antibody, epitope retrieval method, visualization system, method of interpretation, and cut-off level may all affect the level of EGFR expression recorded. This may partly explain the conflicting results on EGFR as a prognostic indicator (Kallio et al. 2003; Merseburger et al. 2005; Nicholson et al. 2001).

However, in NSCLC, EGFR expression has a "weak" association with overall survival (OS). Many studies in NSCLC have reported an association of EGFR to poor survival (Ohsaki et al. 2000; Veale et al. 1993; Volm et al. 1998), while others report a prognostic correlation for EGFR only in combination with other proteins like HER2neu (Brabender et al. 2001). However, in a review of the literature, more studies actually failed to show a significant correlation between EGFR expression and OS (Meert et al. 2002).

In order to determine whether there is any correlation between the level of EGFR expression and OS, exploratory analysis of EGFR expression was performed using diagnostic samples from patients with advanced NSCLC participating in the Phase III INTACT (IRESSA NSCLC Trial Assessing Combination Treatment) trials, which failed to show survival benefit with gefitinib (IRESSA) (Giaccone et al. 2004; Herbst et al. 2004). In these studies, no selection based on EGFR expression was performed because this was not correlated with response to EGFR-tyrosine kinase inhibitors (EGFR-TKIs) in preclinical models (AstraZeneca data on file). However, it might be theoretically possible to find a subgroup of patients with a specific EGFR pattern, who were predicted to benefit significantly from treatment. The exploratory objective was to retrospectively identify prognostic indicators for first-line chemotherapy-treated patients. We aimed to determine the prognostic effect of EGFR in these patients and whether prognostic effects of EGFR were independent of other known clinical prognostic factors.

\section{Materials and methods}

\section{Randomization}

All patients were randomized to standard chemotherapy plus one of the following: gefitinib $250 \mathrm{mg} /$ day, gefitinib $500 \mathrm{mg} /$ day, or placebo. Allocation to the three treatment groups was performed by dynamic randomization (Pocock and Simon 1975) to obtain treatment balance for the following stratification factors: weight loss in the previous 6 months $\leq 5 \%$ versus $>5 \%$; disease stage III versus IV; performance status (PS) of 0 or 1 versus 2 ; or measurable disease versus non-measurable disease.

\section{Patients}

Eligibility criteria have already been reported (Giaccone et al. 2004; Herbst et al. 2004). The INTACT study was performed in accordance with the Declaration of Helsinki, and patients gave an informed consent prior to inclusion in the study (Giaccone et al. 2004; Herbst et al. 2004). Donation of tumor samples was not obligatory for participation in the INTACT studies.

Handling and assessment of tissue samples

NSCLC tissue samples were obtained prior to start of therapy. The samples were formalin-fixed and paraffin-embedded according to prespecified guidelines. All handling of trial material was performed in compliance with Good Clinical and Good Laboratory Practices' guidelines (International Conference on Harmonisation 2001; Organisation for Economic Co-operation and Development 2007). A medical pathologist, blinded to treatment and outcome, assessed sample evaluability, growth pattern, staining patterns, and staining intensities. Due to the inability to evaluate growth pattern, cytology specimens were excluded from the analysis.

\section{EGFR immunohistochemistry}

Tissue specimens were stained by IHC using the validated EGFR pharmDx ${ }^{\text {TM }}$ (Dako, Denmark) assay, which utilizes a standard staining protocol (Dako, EGFR pharmDx ${ }^{\mathrm{TM}}$ product insert) (Spaulding and Spaulding 2002). The EGFR pharmDx ${ }^{\mathrm{TM}}$ assay detects native EGFR as well as mutant EGFR vIII (Andersen et al. 2004). Further, the EGFR phar$\mathrm{mDx}^{\mathrm{TM}}$ assay has been shown not to cross-react with the other three members of the erbB receptor family, erbB2, erbB3, and erbB4 (Andersen et al. 2004; Spaulding and Spaulding 2002). 
Fig. 1 Photomicrographs of squamous cell carcinoma $(S C)$ $(\mathbf{a}-\mathbf{c})$ and well differentiated adenocarcinoma $(A C)(\mathbf{d}-\mathbf{f})$ immunostained with the Dako (EGFR) pharmDx ${ }^{\mathrm{TM}}$ assay. In $\mathrm{SC}$ the growth pattern is solid. When the entire circumference of the cell membrane is stained positive, the impression of the tumor is that of chicken-wire or fishnet. In AC the growth pattern is glandular, allowing for a basolateral staining when the entirety of the membrane is stained. The cell membrane may have different staining intensities: $\mathbf{a}$ and $\mathbf{d}$ weak $1+$ staining; $\mathbf{b}$ and $\mathbf{e}$ moderate $2+$ staining; $\mathbf{c}$ and $\mathbf{f}$ strong $3+$ staining
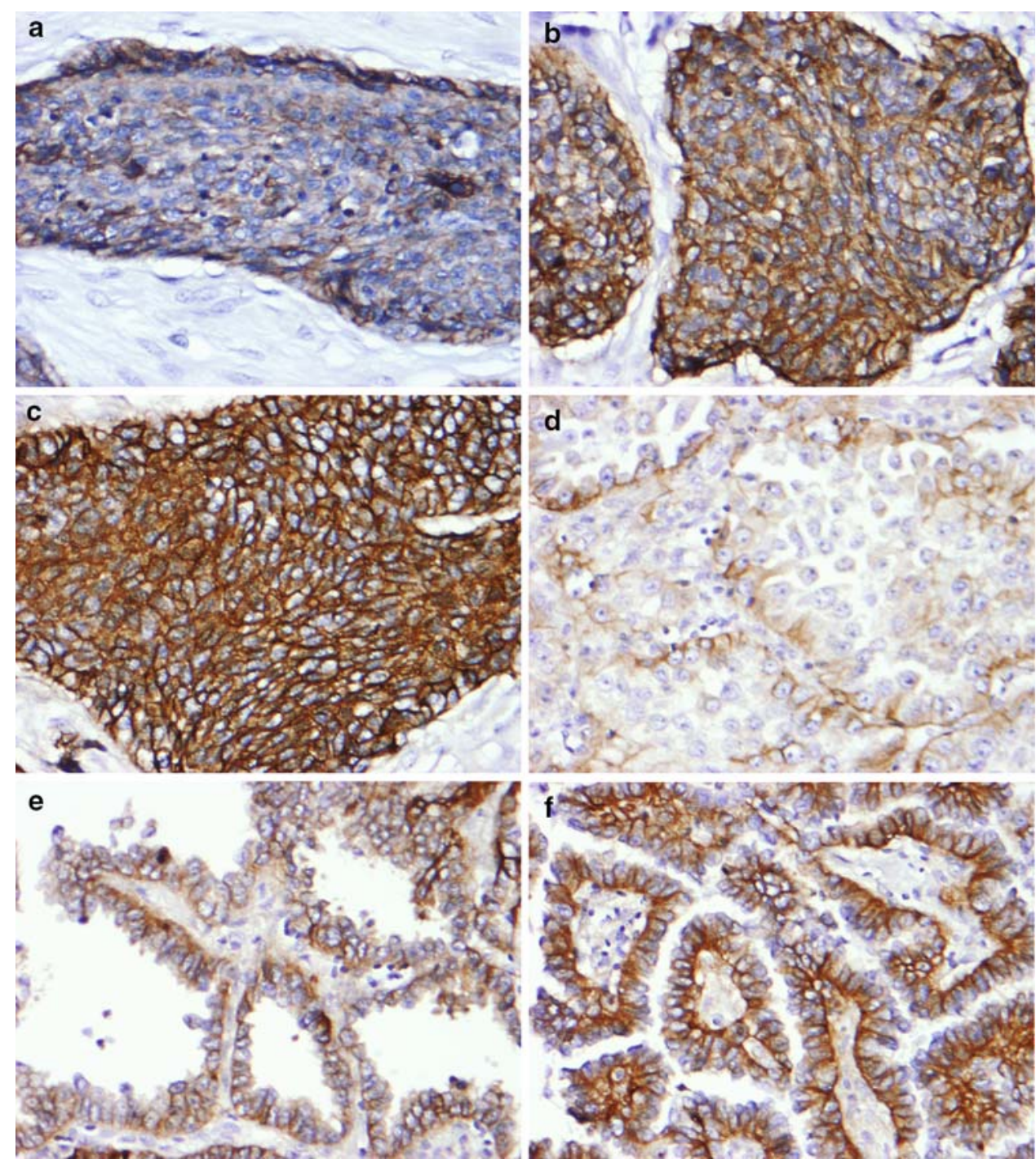

\section{Capture of EGFR staining intensities and growth pattern}

The pattern of membrane and cytoplasmic staining was assessed in each tumor sample. The sum of the percentages of tumor cells was recorded with four levels of staining intensity (no staining [0], weak $[1+]$, moderate $[2+]$, or strong staining $[3+]$ ) totaling $100 \%$ (Fig. 1a-f). This was true for both membrane and cytoplasmic staining.

The medical pathologist (MJ) categorized the growth pattern in three categories: (1) solid, (2) glandular, or (3) mixed solid and glandular. (1) For tumors with solid growth pattern, as seen in squamous cell carcinoma, or poorly differentiated adenocarcinoma (AC), complete staining of the membrane resulted in a staining pattern, figuratively described as chicken-wire or fishnet staining (Fig. 1a-c). (2) The glandular growth pattern reflects the growth pattern of a well-differentiated AC, including, but not restricted to, the growth of bronchioloalveolar carcinoma (BAC). The maximal membrane staining in well- differentiated $\mathrm{AC}$ is found in the basal and lateral membranes, mirroring the fact that tumor cells of $\mathrm{AC}$ with glandular growth are polarized (Fig. 1d-f). (3) Tumors with mixed growth pattern (adenosquamous carcinoma) may contain areas with both chicken-wire basolateral-membrane staining. The rationale for capturing complete and basolateral-membrane staining stems from the established scoring system for HercepTest ${ }^{\mathrm{TM}}$ (Dako, Denmark), where complete membrane staining in $>10 \%$ of tumor cells determines eligibility for trastuzumab treatment (Slamon et al. 1989).

\section{Statistical analysis}

Both the predictive and the prognostic effects of EGFR were investigated.

An exploratory analysis on OS was performed independently for patients randomized to placebo and to gefitinib ( 250 or $500 \mathrm{mg} /$ day), as well as for combined treatment groups. Analyses were stratified by trial. A restricted 
backwards elimination Cox proportional hazard regression analysis, by which only biologically relevant eliminations took place in each step, was conducted. In each step, the statistical model contains a term for up to three variables (intensity level $1+, 2+$, and $3+$ ) within each of three categories: (a) membrane, (b) complete or basolateral, and (c) cytoplasmic staining. Tests are restricted to coefficients of all pairs of adjacent variables within the same category being equal. For example, the test of the coefficient to the variable at the lowest level (1+) being equal to zero is considered as a test for collapsing this level with the no staining level. Additionally, let us assume that in a previous step the hypothesis that the effect of staining being at level +1 and +2 is accepted as being equal, leading to the adjoining of these two levels, then testing that the coefficient to the adjoined level is equal to zero is identical to testing whether the adjoined level $(1+$ and $2+)$ could be further combined with the no staining level, and thereby removed from the model. In each step the test producing the largest $P$ value determines the candidate to be adjoined next.

The analyses were conducted to identify categorical and continuous EGFR factors (growth pattern, membrane, and cytoplasmic staining intensity and completeness of membrane staining) prognostic for survival within the group of placebo and gefitinib-treated patients, separately as well as combined. Variables found to have a statistically significant prognostic effect within at least one of the two treatment groups were also tested for treatment interaction, to determine if they served as predictive factors.

It was further analyzed whether EGFR-related variables, found to be statistically significant prognostic factors, had an effect of their own, independent of other potential prognostic factors such as gender, PS, stage, histology, and site of metastasis. This was done by repeating the above-mentioned procedure based on the EGFR-related variables in combination with the potential clinical prognostic factors.

Tests were performed at the $10 \%$ two-sided significance level (i.e., $P<0.10$ was considered statistically significant).

\section{Results}

Demographics

A total of 631 patients had tumor samples available and provided consent to this exploratory part of the INTACT studies. Tissue samples that were fully evaluable for EGFR were available for 516 patients ( 219 and 297 from INTACT 1 and 2 , respectively).

Comparison of the demographic factors and patient disease characteristics such as histology, PS, stage, and site of metastasis revealed that this patient population, with a tumor sample fully evaluable for EGFR, was similar to that of both INTACT trials from whom tumor samples were less than fully evaluable for EGFR or not evaluable, and representative of the full INTACT population (data on file).

Prognostic and predictive effect

\section{Prognostic effect}

The two EGFR-related variables of growth pattern and membrane staining were found to have statistically significant prognostic effects, both in patients treated with gefitinib $(P=0.0023)$ and placebo $(P=0.0128)$, as well as in the two groups combined $(P<0.0001)$ (Table 1$)$.

There was no statistically significant difference in OS between patients having tumors with a glandular growth pattern and those having tumors with a mixed growth pattern in either of the treatment groups (data not shown). Therefore, these patients (glandular/mixed) were combined and will subsequently be referred to as "glandular patients". The others will be referred to as "solid patients". Glandular patients had statistically significantly better survival than solid patients when the two treatment groups were combined $(P=0.0001)$ or considered separately (placebo, $P=0.0220$; gefitinib, $P=0.0014$ ) (Table 1). This prognostic effect was of the same magnitude in both treatment groups.

The total percentage of cytoplasmic staining and the total percentage of membrane staining were highly correlated $(r=0.92)$. EGFR expression, as measured by membrane staining, had the strongest prognostic effect of the two, and conditioned on this effect, cytoplasmic staining had no prognostic effect of its own. All further analyses were consequently focused on membrane staining. The percentage of membrane staining had a statistically significant prognostic effect as a continuous covariate in both treatment groups (placebo, $P=0.0147$; gefitinib, $P=0.0463$ ) and in both groups combined $(P=0.0025)$ (Table 1$)$. In the placebo group, the most statistically significant variable was membrane staining of weak $(1+)$, moderate $(2+)$ or strong (3+) intensity, whereas in the gefitinib group it was the variable "membrane staining of moderate $(2+)$ or strong (3+) intensity". According to the prespecified procedure, two variables, "membrane staining of weak $(1+)$ " and "moderate $(2+)$ or strong (3+)", were analyzed based on both treatment groups combined, resulting in identifying membrane staining of weak $(1+)$, moderate $(2+)$, or strong (3+) intensity as the most statistically significant variable. As membrane staining is a continuous covariate, the effect of membrane staining is a trend effect. The hazard ratio (HR) is calculated based on membrane staining of 0 or $100 \%$, only.

The prognostic effect of both EGFR-related variables (growth pattern and membrane staining) is of the same size 
Table 1 Prognostic effects of EGFR variables

\begin{tabular}{|c|c|c|c|c|c|}
\hline Analysis based on & Solid N/E & Glandular N/E ${ }^{a}$ & Factor & $P$ value & HR (95\% CIs) \\
\hline Placebo group & \multirow[t]{3}{*}{$108 / 80$} & \multirow[t]{3}{*}{$56 / 34$} & Glandular/solid & (1) 0.0220 & $0.604(0.392-0.93)$ \\
\hline Placebo group & & & $100 \% / 0 \%$ Membrane staining & (2) 0.0147 & $0.521(0.309-0.880)$ \\
\hline Placebo group & & & Both & (*) 0.0128 & \\
\hline Gefitinib group & \multirow[t]{3}{*}{$223 / 161$} & \multirow[t]{3}{*}{$129 / 74$} & Glandular/solid & (1) 0.0014 & $0.628(0.472-0.835)$ \\
\hline Gefitinib group & & & $100 \% / 0 \%$ Membrane staining & (3) 0.0463 & $0.687(0.475-0.994)$ \\
\hline Gefitinib group & & & Both & (*) 0.0023 & \\
\hline Placebo and gefitinib groups & \multirow[t]{3}{*}{$331 / 241$} & \multirow[t]{3}{*}{$185 / 108$} & Glandular/solid & (1) 0.0001 & $0.631(0.499-0.798)$ \\
\hline Placebo and gefitinib groups & & & $100 \% / 0 \%$ Membrane staining & (2) 0.0025 & $0.642(0.482-0.856)$ \\
\hline Placebo and gefitinib groups & & & Both & $(*)<0.0001$ & \\
\hline \multirow{2}{*}{$\begin{array}{l}\text { Both groups conditioned on effect of } \\
\text { other prognostic factors }\end{array}$} & \multirow[t]{2}{*}{$329 / 240$} & \multirow[t]{2}{*}{$184 / 108^{\mathrm{b}}$} & & (1) 0.0011 & $0.668(0.525-0.850)$ \\
\hline & & & & (2) 0.0158 & $0.694(0.516-0.934)$ \\
\hline
\end{tabular}

${ }^{a}$ Number of subjects $(\mathrm{N}) /$ number of events (E). Event is death

b Missing values for prognostic factors in three cases

* Simultaneous test of both growth pattern and membrane staining

$P$-values are for the null hypothesis (1) of no effect of growth pattern group and (2) of no effect of membrane staining of weak (1+) or moderate $(2+)$ or strong (3+) intensity. Because membrane staining of weak intensity (1+) did not show statistical significance in the gefitinib group the $P$ value in this group is for the null hypothesis (3) no effect of membrane staining of moderate (2+) or strong (3+) intensity

in terms of HR, consistent over treatment groups and independent of other prognostic factors (Table 1). A further exploration of the trend effect is shown in Fig. 2a (solid growth pattern) and $\mathrm{b}$ (glandular growth pattern), which show the median survival times predicted by the Cox proportional hazard model, incorporating membrane staining as a continuous covariate, i.e., a trend effect of membrane staining. As shown in Fig. 2a and b, with many groups containing small numbers of patients, it was expected that the variability of the estimated medians around the predicted trend was large, indicated by the wide confidence intervals (CIs). In all cases, where both upper and lower 99\% confidence limits could be estimated, the interval included the median time predicted by the Cox proportional hazard trend model, suggesting a reasonable model fit. However, a lack of fit test rejected the null hypothesis that the data fitted the Cox proportional hazard trend model $(P=0.0240)$. The lack of fit may be caused by deviation from the trend model, or because of a lack of proportional hazard.

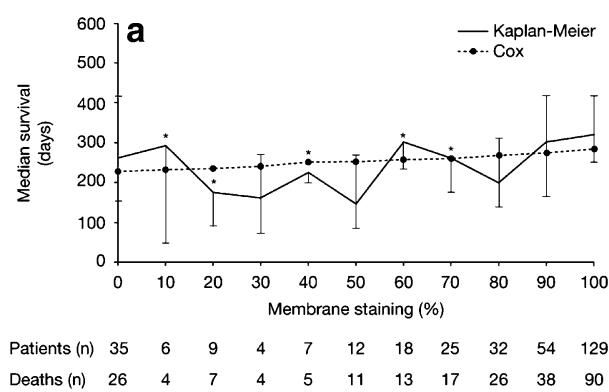

Fig. 2 a Estimated median survival times for patients with tumors having a solid growth pattern (including 99\% CIs). *Estimation not possible. b Estimated median survival times for patients with tumors
The prognostic trend effect appears to be more pronounced in patients having a glandular growth pattern (Fig. 2b) than in those having a solid growth pattern (Fig. 2a). Since the interaction effect between growth pattern and percentage membrane staining was not statistically significant $(P=0.1474)$, the prognostic trend effect may be the same for all patients. In spite of this, the prognostic trend effect in the glandular growth pattern group was highly statistically significant $(P=0.0035)$, whereas the trend effect in the solid growth pattern group was not statistically significant $(P=0.1094)$, yet another possibility is that percentage membrane staining only has a prognostic trend effect in the group of patients not having a solid growth pattern.

\section{Prognostic effect using alternative scoring method}

An additional post-hoc analysis of the EGFR expression data from the INTACT trials was conducted, to facilitate a

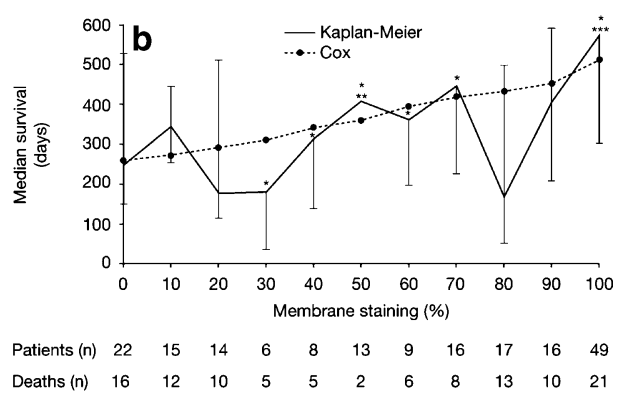

having a glandular growth pattern (including $99 \%$ CIs). *Estimation not possible; **estimation not possible: 406 days inserted in figures; ***estimation not possible: 573 days inserted in figures 
comparison between the data presented here, and the results of the erlotinib BR21 trial (Shepherd et al. 2005). In this analysis, EGFR-positive was defined as $\geq 10 \%$ of cells staining positive for EGFR. Using this scoring system, 459 (89\%) and $57(11 \%)$ patients were found to be EGFR-positive and EGFR-negative, respectively. When both treatments groups were combined, the median survival for the EGFR-positive group was 307 days with 261 days for the EGFR-negative group; the HR comparing the positive to negative groups was $0.812(95 \% \mathrm{CI}=0.588-1.123)$, suggesting similar results to the original analysis that patients with EGFR expression have a better prognosis in this study. To look for a predictive effect, the gefitinib treatment group contained 312 EGFR-positive and 40 EGFR-negative classed patients with a median survival 296 and 218 days, respectively. An HR of 0.959 (95\% CI $=0.635-1.449)$ was estimated in Cox regression analysis with a $P$ value of 0.84 .

\section{Comparison with other clinical prognostic factors}

To illustrate how strong the prognostic effect of the two EGFR-related variables is (growth pattern and staining intensity), the effect of these variables was compared with other well-known clinical prognostic factors.

The strength of the effect of the EGFR prognostic factor related to growth pattern expressed as an HR (solid vs. glandular) was $0.668(95 \% \mathrm{CI}=0.525-0.850)$ (Table 1). This was comparable to the strength of the effect of other well-known clinical prognostic factors such as disease stage (HR for stage IV vs. stage III: 0.695 [95\% CI = 0.523-0.923]), liver metastasis (HR for present vs. not present: 0.718 [95\% CI $=0.546-0.946]$ ), and bone metastasis (HR for present vs. not present: $0.730[95 \% \mathrm{CI}=0.566-$
0.941]). Only the effect of PS appeared to be stronger (HR for ' 2 ' vs. '0 or 1': 0.484 [95\% CI $=0.351-0.667]$ ). The strength of the prognostic effect of membrane staining at weak (1+), moderate $(2+)$, or strong (3+) intensity, when shifting from 0 to $100 \%$, was similar. This maximum shift from 0 to $100 \%$ membrane staining gave a $30 \%$ improvement in survival (HR 0.694; 95\% CI $=0.516-0.934)$ (Table 1).

To further illustrate the strength of the prognostic effect of the two EGFR-related variables, the Kaplan-Meier plots for growth pattern (Fig. 3a) and membrane staining (Fig. 3b) were compared with those for disease stage III versus IV (Fig. 3c), as stage of disease showed the strongest prognostic effect in the original INTACT efficacy analysis.

\section{Relationship between growth pattern and histology}

Because it was expected that histology and growth pattern were at least partially confounded, it was further investigated whether growth pattern had an independent effect which could not be explained by the recorded histology on the original six-category scale. This was done in spite of the fact that the dichotomized histology variable (AC [including $\mathrm{BAC}$ ] vs. other histologies) did not have a statistically significant prognostic effect on survival.

Since all BACs had a glandular growth pattern, it was not possible to distinguish an effect of histology and growth pattern. BAC, which is a subgroup of AC, was therefore excluded when investigating dependence between histology and growth pattern (14 patients with eight deaths).

The analysis showed that the effect of the two EGFR prognostic factors (growth pattern and membrane staining)
Fig. 3 a Prognostic effect of growth pattern illustrated by Kaplan-Meier plot for survival. b Prognostic effect of membrane staining illustrated by KaplanMeier plot for survival. c Prognostic effect of disease stage illustrated by Kaplan-Meier plot for survival
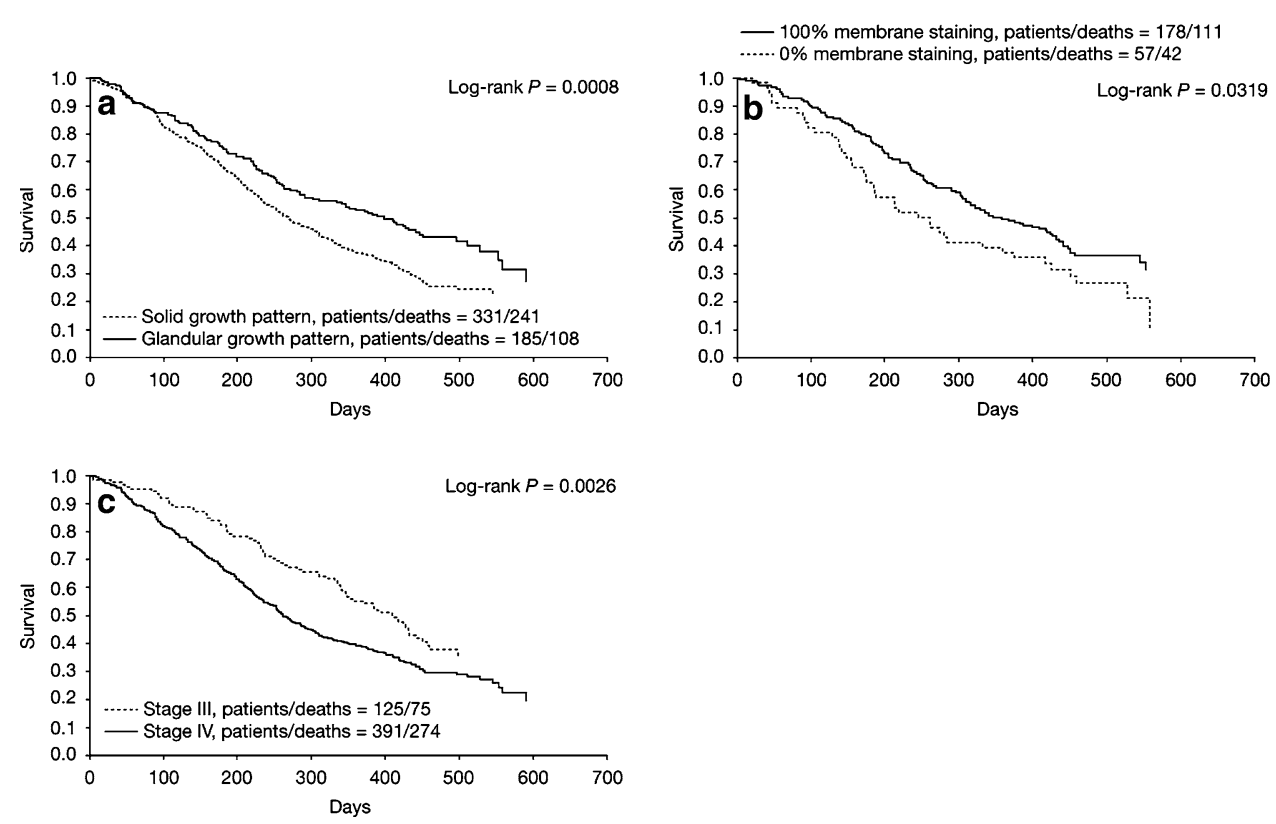
was statistically significant in the group of patients with $\mathrm{AC}$ excluding BAC ( $P=0.0019$ and $P=0.0137$, respectively). The strength of the effect of the two EGFR prognostic factors was similar to that observed when all (516) specimens were analyzed (data not shown). It is not relevant to test the effect of growth pattern in the group of non ACs. The effect of membrane staining was more pronounced in the AC group than in the heterogeneous group of patients with other histologies (excluding BAC), HRs being 0.60 (95\% $\mathrm{CI}=0.40-0.90)$ and $0.75(95 \% \mathrm{CI}=0.47-1.17)$, respectively.

\section{Relationship between growth pattern and histology to mutation and amplification of EGFR}

A post-hoc analysis was conducted to look at the effect of EGFR mutation and amplification status on the results seen for staining intensity and growth pattern in a subset of patients also looked at in the recent report by Bell et al. (2005) (Bell et al. 2005). Of the 516 patients in the analysis of staining intensity and growth pattern, 246 (47.7\%) had sufficient DNA for EGFR mutation testing and 387 (75\%) had sufficient DNA for measurement of gene amplification by DNA sequencing. For EGFR mutation, 25 (20.2\%) patients were found to contain an EGFR mutation while 225 were classified as mutation-negative, leaving 270 with missing EGFR-mutation status. For EGFR amplification, $25(6.4 \%)$ patients had EGFR gene amplification, 362 (93.6\%) were EGFR amplification-negative, with 129 classified as "missing".

For the statistical analysis, three groups were considered, "positive" (either defined as containing mutation or amplification in the respective analysis), "negative" or "missing". Amplification was found to have no effect or correlation with either staining intensity or growth pattern, and also had no prognostic effect alone in a model with both treatments combined. For the analysis of EGFR mutation in the model with staining intensity and growth pattern, EGFR mutation was found to be prognostic $(P=0.03)$, and both staining intensity $(P=0.007)$ and growth pattern $(P=0.0005)$ maintained significant prognostic effects as well.
Predictive effect

Neither the growth pattern nor the EGFR membrane staining variable had a statistically significant predictive effect. This was illustrated in an analysis of a model including treatment, growth pattern (solid vs. glandular group) and membrane staining, together with the interactions between treatment and growth pattern, and treatment and membrane staining. The $P$ values for the test of interactions in this model were $P=0.8688$ for treatment and growth pattern, and $P=0.4967$ for treatment and membrane staining. Based on this model, including the effects of the two interactions, the HR and corresponding 95\% CI between gefitinib and placebo were estimated for four populations: each of the growth patterns, solid and glandular, with either 0 or $100 \%$ membrane staining (Table 2). The wide CIs within each of the four populations indicate that even though no statistically significant predictive effect was found, the sample sizes were not large enough to exclude the existence of a clinically significant predictive effect.

\section{Discussion}

The results from the current analysis of the two large, Phase III INTACT 1 and 2 studies represent the largest analysis of EGFR expression and OS in patients with NSCLC to date. The INTACT results provide highly statistically significant evidence that EGFR expression, as measured by percentage-membrane staining using the EGFR pharmDx ${ }^{\mathrm{TM}}$ assay, is a strong independent prognostic indicator for improved survival in patients treated with platinum-based chemotherapy with tumors that exhibit glandular growth pattern. It may be speculated whether the prognostic trend effect found in the present studies reflects a true prognostic effect of EGFR staining in tumors with glandular growth pattern, or whether these tumors respond better to first-line platinum-based chemotherapy.

The prognostic trend effect of percentage-membrane staining in patients with a solid growth pattern is more uncertain, ranging from no effect to the same effect as for patients with a glandular growth pattern (Bingle et al. 2005).

Table 2 Estimates of the effect of treatment (predictive effect) in four different groups defined by EGFR variables

\begin{tabular}{llrr}
\hline Definition of group & N/E Gefitinib & N/E Placebo & HR $^{\mathrm{b}}(95 \%$ CIs $)$ \\
\hline Solid growth pattern and membrane staining $=0 \%$ & $23 / 15$ & $12 / 11$ & $0.876(0.522-1.471)$ \\
Glandular growth pattern and membrane staining $=0 \%$ & $17 / 11$ & $5 / 5$ & $0.840(0.496-1.423)$ \\
Solid growth pattern and membrane staining $=100 \%$ & $86 / 60$ & $43 / 30$ & $1.087(0.786-1.504)$ \\
Glandular growth pattern and membrane staining $=100 \%$ & $38 / 15$ & $11 / 6$ & $1.042(0.632-1.719)$ \\
\hline
\end{tabular}

\footnotetext{
${ }^{a}$ Number of subjects $(\mathrm{N}) /$ number of events (E). Event is death

${ }^{\mathrm{b}} \mathrm{HR}$, hazard ratio for treatment effect (gefitinib/placebo)
} 
In this analysis, patients with tumors exhibiting areas of well-differentiated AC (i.e., glandular growth pattern comprised of AC, BAC, and adenosquamous carcinomas) had better survival than those with tumors that had a solid growth pattern.

In addition to prognostic factors, which have effects on survival irrespective of treatment, much research is being undertaken in an attempt to identify factors that may predict response to treatment. The results from the present analysis showed that neither growth pattern nor EGFR-membrane staining had a statistically significant predictive effect. However, the study may have been limited by power, given that tumor samples were not mandatory and were only obtained from less than one-third of patients. Furthermore, in these INTACT studies, gefitinib was in combination with chemotherapy and any gefitinib-treatment effect may have been masked by the combination. Additionally, given the overall result of both trials, in which addition of gefitinib to chemotherapy did not result in superior efficacy, a predictive effect is less likely to be seen.

Results from the TALENT (Tarceva Responses in Conjunction with Cisplatin and Gemcitabine) and TRIBUTE (Tarceva Responses in Conjunction with Paclitaxel and Carboplatin) studies demonstrated that the addition of another EGFR-TKI, in this case erlotinib, to standard chemotherapy regimens also did not improve outcome for chemotherapy-naive patients with advanced NSCLC (Herbst et al. 2005). As with INTACT 1 and 2, and gefitinib, the level of EGFR expression in both TALENT and TRIBUTE were not predictive of responses to erlotinib (Gatzemeier et al. 2007; Herbst et al. 2004, 2005).

Exploratory analysis of 157 tumor biopsies from patients participating in the Phase II IDEAL (IRESSA Dose Evaluation in Advanced Lung cancer) trials revealed that tumor EGFR-membrane staining is not clinically relevant for predicting response in patients receiving gefitinib monotherapy for pretreated advanced NSCLC (Bailey et al. 2003).

In the Phase III ISEL (IRESSA Survival Evaluation in Lung cancer) trial that failed to demonstrate an overall survival of gefitinib as compared to placebo in patients who had failed chemotherapy (Thatcher et al. 2005), analysis of tumor biopsy samples demonstrated that EGFR protein expression-positive patients achieved significantly better survival with gefitinib versus placebo than patients with EGFR protein expression-negative tumors (interaction test for EGFR protein expression-positive vs. EGFR protein expression-negative patients, $P=0.049)$. Better response rates were also reported for EGFR protein expression-positive patients than EGFR protein expression-negative patients (8.2 vs. 1.5\%) (Hirsch et al. 2006). Furthermore, results from the randomized, placebo-controlled trial of erlotinib in patients with locally advanced or metastatic
NSCLC after failure of at least one chemotherapy regimen (BR21), suggest a correlation between EGFR expression and survival (Shepherd et al. 2005; Tsao et al. 2005). Erlotinib prolonged survival in the EGFR-positive subgroup ( $n=184$; HR $0.68 ; 95 \% \mathrm{CI}=0.49-0.95 ; P=0.02$ ), but did not appear to have an effect on survival in the EGFR-negative subgroup ( $n=141$; HR $0.93 ; 95 \% \mathrm{CI}=0.63-1.36$; $P=0.70)$.

As EGFR expression was measured using a different scoring method in the erlotinib BR21 trial from that used in this analysis of EGFR in the INTACT trials, an additional post-hoc analysis (data not shown) was conducted looking at the effect of EGFR expression with the same definition, from BR21 ( $\geq 10 \%$ of cells staining positive equals EGFRpositive). This analysis suggested no statistically significant differences between EGFR-positive and -negative patients, when treated with gefitinib in combination with chemotherapy.

Emerging data indicate that mutations in the EGFR gene are associated with dramatic responses to gefitinib (Lynch et al. 2004; Paez et al. 2004; Sordella et al. 2004). Mutation analysis of samples from INTACT 1 and 2 did not show a statistically significant difference in response to gefitinib plus chemotherapy according to EGFR genotype (Bell et al. 2005). OS was not affected by the addition of gefitinib to chemotherapy in patients with EGFR mutations $(\mathrm{HR}=1.77 ; 95 \%=$ CI 0.50-6.23). However, patients with EGFR-mutation-positive tumors treated with chemotherapy alone had a better OS compared to patients with mutationnegative tumors (median OS, 19.4 vs. 9.2 months; $\mathrm{HR}=0.48 ; 95 \% \mathrm{CI}=0.29-0.82$ ), raising the possibility that this genetically defined subset of NSCLC may have a more favorable natural history, and that EGFR mutations may also serve as a prognostic factor. Our analysis of a subset of these same patients included in the current analysis finds similar results for the prognostic effect.

As in other reports, mutational status of tumors from BR21 was shown to possibly increase responsiveness to erlotinib. The response rate among patients with mutations was twice that among patients with wild-type EGFR, although the difference was not significant, perhaps because the number of responses was small: $7 \%$ of those with wildtype EGFR had a response compared with $16 \%$ of those with an EGFR mutation $(P=0.37)$ (Tsao et al. 2005). Importantly however, the risk of death did not differ significantly among patients with EGFR mutations who received erlotinib compared with patients who received placebo (HR $=0.77 ; 95 \%$ CI $=0.40-1.50 ; P=0.54)$, or among patients with wild-type EGFR who received erlotinib compared with such patients who received placebo $(\mathrm{HR}=0.73$; 95\% CI $=0.49-1.10 ; P=0.13)$.

In a recent report from Eberhard et al. (Eberhard et al. 2005; Kishi et al. 2005), EGFR mutations were found in 
$13 \%$ of tumor samples from patients treated in the TRIBUTE study (Herbst et al. 2005) and was associated with longer survival irrespective of treatment $(P<0.001)$. This data suggests that EGFR mutation is prognostic of longer survival rather than predictive. However, among patients treated with erlotinib, EGFR mutations were associated with a better response rate and trend towards better time to progression but no survival increase $(P<0.05,0.092$ and 0.96 , respectively). On the other hand, K-ras mutations were associated with a significant decrease in time to progression and survival in patients treated with erlotinib.

Many other biomarkers are under investigation as predictive factors, for example, the components of the Akt and MAPK signaling pathways. Indeed, patients with tumors that were positive for phosphorylated Akt (pAkt) have been shown to have a better response rate, disease control rate, and time to progression with gefitinib than those that were negative (Cappuzzo et al. 2004). The data from 78 patients treated in Japan with gefitinib showed that pAkt was not predictive of response (Kishi et al. 2005).

Further work has investigated the relationship between EGFR gene copy number (assessed by fluorescence in situ hybridization [FISH]), EGFR mutations, EGFR protein expression and Akt activation status as predictive markers for gefitinib therapy in advanced NSCLC (Cappuzzo et al. 2005). In a multivariable analysis, high EGFR gene copy number was significantly associated with better survival $(\mathrm{HR}=0.44 ; 95 \% \mathrm{CI}=0.23-0.82)$, indicating that this may be a molecular predictor for gefitinib efficacy in advanced NSCLC. The analysis of tumor biopsy samples from patients participating in the ISEL trial has also demonstrated that high EGFR gene copy number was a predictor of increased survival with gefitinib (comparison of HRs high vs. low copy number, $P=0.045$ ) (Hirsch et al. 2006). Amplification of EGFR was associated with responsiveness to erlotinib $(P=0.03)$ in tumor samples from BR21 subjected to FISH (Tsao et al. 2005).

The current analysis in first-line, platinum-based, chemotherapy-treated patients with NSCLC provides statistically significant evidence that the combination of EGFR expression and growth pattern is a strong prognostic indicator for improved survival within this setting.

The prognostic trend effect of percentage membrane staining in patients with a solid growth pattern is more uncertain, ranging from no effect to the same effect as for patients with a glandular growth pattern. In this analysis, patients with tumors exhibiting areas with well-differentiated AC (i.e., glandular growth pattern) had better survival than those with tumors that had a solid growth pattern. This is in agreement with earlier studies, which have shown that NSCLC patients with well-differentiated tumors survive longer than patients with poorly differentiated tumors (Gawrychowski et al. 2003; Nakayama et al. 1997).
In summary, many factors may have contributed to the lack of a survival benefit for gefitinib in combination with first-line platinum-based chemotherapy. The debate continues on the usefulness of EGFR expression to help select patients for treatment. The combination of EGFR expression and growth pattern may be a strong prognostic indicator for improved survival, while high EGFR gene copy number and EGFR mutations could be associated with better response to EGFR-TKIs.

Acknowledgments We thank Mark Walker Ph.D. from Complete Medical Communications who provided medical writing support funded by AstraZeneca.

Open Access This article is distributed under the terms of the Creative Commons Attribution Noncommercial License which permits any noncommercial use, distribution, and reproduction in any medium, provided the original author(s) and source are credited.

\section{References}

Andersen FG, Jensen SS, Pii K, Spaulding BO (2004) Characterization of a new monoclonal antibody, clone $2-18 \mathrm{C} 9$, for the measurement of epidermal growth factor receptor expression in solid tumours. Proc Am Assoc Cancer Res, vol 45

Bailey LR, Kris M, Wolf M, Kay A, Averbuch S, Askaa J, Janas M, Schmidt K, Fukuoka M (2003) Tumor EGFR membrane staining is not clinically relevant for predicting response in patients receiving gefitinib ('Iressa', ZD1839) monotherapy for pretreated advanced non-small-cell lung cancer: IDEAL 1 and 2. Proc Am Assoc Cancer Res, vol 44 (2nd edn):1362, abs LB-170

Bell DW, Lynch TJ, Haserlat SM, Harris PL, Okimoto RA, Brannigan BW, Sgroi DC, Muir B, Riemenschneider MJ, Iacona RB, Krebs AD, Johnson DH, Giaccone G, Herbst RS, Manegold C, Fukuoka M, Kris MG, Baselaga J, Ochs JS, Haber DA (2005) Epidermal growth factor receptor mutations and gene amplification in nonsmall-cell lung cancer: molecular analysis of the IDEAL/INTACT gefitinib trials. J Clin Oncol 23:8081-8092

Bingle L, Cross SS, High AS, Wallace WA, Devine DA, Havard S, Campos MA, Bingle CD (2005) SPLUNC1 (PLUNC) is expressed in glandular tissues of the respiratory tract and in lung tumours with a glandular phenotype. J Pathol 205:491-497

Brabender J, Danenberg KD, Metzger R, Schneider PM, Park J, Salonga D, Holscher AH, Danenberg PV (2001) Epidermal growth factor receptor and HER2-neu mRNA expression in non-small cell lung cancer is correlated with survival. Clin Cancer Res 7:1850-1855

Cappuzzo F, Hirsch FR, Rossi E, Bartolini S, Ceresoli GL, Bemis L, Haney J, Witta S, Danenberg K, Domenichini I, Ludovini V, Magrini E, Gregorc V, Doglioni C, Sidoni A, Tonato M, Franklin WA, Crino L, Bunn PA Jr, Varella-Garcia M (2005) Epidermal growth factor receptor gene and protein and gefitinib sensitivity in non-small-cell lung cancer. J Natl Cancer Inst 97:643-655

Cappuzzo F, Magrini E, Ceresoli GL, Bartolini S, Rossi E, Ludovini V, Gregorc V, Ligorio C, Cancellieri A, Damiani S, Spreafico A, Paties CT, Lombardo L, Calandri C, Bellezza G, Tonato M, Crinò L (2004) Akt phosphorylation and gefitinib efficacy in patients with advanced non-small-cell lung cancer. J Natl Cancer Inst 96:1133-1141

Eberhard DA, Johnson BE, Amler LC, Goddard AD, Heldens SL, Herbst RS, Ince WL, Janne PA, Januario T, Johnson DH, Klein P, Miller VA, Ostland MA, Ramies DA, Sebisanovic D, Stinson JA, Zhang YR, Seshagiri S, Hillan KJ (2005) Mutations in the 
epidermal growth factor receptor and in KRAS are predictive and prognostic indicators in patients with non-small-cell lung cancer treated with chemotherapy alone and in combination with erlotinib. J Clin Oncol 23:5900-5909

Gatzemeier U, Pluzanska A, Szczesna A, Kaukel E, Roubec J, De Rosa F, Milanowski J, Karnicka-Mlodkowski H, Pesek M, Serwatowski P, Ramlau R, Janaskova T, Vansteenkiste J, Strausz J, Manikhas GM, von Pawel J (2007) Phase III study of erlotinib in combination with cisplatin and gemcitabine in advanced nonsmall-cell lung cancer: the Tarceva Lung Cancer Investigation Trial. J Clin Oncol 25:1545-1552

Gawrychowski J, Gabriel A, Lackowska B (2003) Heterogeneity of stage IIIA non-small cell lung cancers (NSCLC) and evaluation of late results of surgical treatment. Eur J Surg Oncol 29:178-184

Giaccone G, Herbst RS, Manegold C, Scagliotti G, Rosell R, Miller V, Natale RB, Schiller JH, von Pawel J, Pluzanska A, Gatzemeier U, Grous J, Ochs JS, Averbuch SD, Wolf MK, Rennie P, Fandi A, Johnson DH (2004) Gefitinib in combination with gemcitabine and cisplatin in advanced non-small-cell lung cancer: a phase III trial-INTACT 1. J Clin Oncol 22:777-784

Herbst RS, Giaccone G, Schiller JH, Natale RB, Miller V, Manegold C, Scagliotti G, Rosell R, Oliff I, Reeves JA, Wolf MK, Krebs AD, Averbuch SD, Ochs JS, Grous J, Fandi A, Johnson DH (2004) Gefitinib in combination with paclitaxel and carboplatin in advanced non-small-cell lung cancer: a phase III trial-INTACT 2. J Clin Oncol 22:785-794

Herbst RS, Prager D, Hermann R, Fehrenbacher L, Johnson BE, Sandler A, Kris MG, Tran HT, Klein P, Li X, Ramies D, Johnson DH, Miller VA (2005) TRIBUTE: a phase III trial of erlotinib hydrochloride (OSI-774) combined with carboplatin and paclitaxel chemotherapy in advanced non-small-cell lung cancer. J Clin Oncol 23:5892-5899

Hirsch FR, Varella-Garcia M, Bunn PA Jr, Franklin WA, Dziadziuszko R, Thatcher N, Chang A, Parikh P, Rodrigues Pereira J, Ciuleanu T, von Pawel J, Watkins C, Flannery A, Ellison G, Donald E, Knight L, Parums D, Botwood N, Holloway B (2006) Molecular predictors of outcome with gefitinib in a Phase III placebocontrolled study in advanced non-small-cell lung cancer. J Clin Oncol 24:5034-5042

International Conference on Harmonisation (2001) ICH harmonised tripartite guideline: guideline for good clinical practice. J Postgrad Med 47:199-203

Kallio JP, Hirvikoski P, Helin H, Kellokumpu-Lehtinen P, Luukkaala T, Tammela TL, Martikainen PM (2003) Membranous location of EGFR immunostaining is associated with good prognosis in renal cell carcinoma. Br J Cancer 89:1266-1269

Kishi K, Homma S, Miyamoto A, Sakamoto S, Motoi N, Yoshimura K (2005) Factors predicting the efficacy of gefitinib in patients with advanced non-small cell lung cancer (NSCLC). J Clin Oncol (Meeting Abstracts) 23:644s, abs 7095

Lynch TJ, Bell DW, Sordella R, Gurubhagavatula S, Okimoto RA, Brannigan BW, Harris PL, Haserlat SM, Supko JG, Haluska FG, Louis DN, Christiani DC, Settleman J, Haber DA (2004) Activating mutations in the epidermal growth factor receptor underlying responsiveness of non-small-cell lung cancer to gefitinib. N Engl J Med 350:2129-2139

Meert AP, Martin B, Delmotte P, Berghmans T, Lafitte JJ, Mascaux C, Paesmans M, Steels E, Verdebout JM, Sculier JP (2002) The role of EGF-R expression on patient survival in lung cancer: a systematic review with meta-analysis. Eur Respir J 20:975-981

Merseburger AS, Hennenlotter J, Simon P, Kruck S, Koch E, Horstmann M, Kuehs U, Kufer R, Stenzl A, Kuczyk MA (2005) Membranous expression and prognostic implications of epidermal growth factor receptor protein in human renal cell cancer. Anticancer Res 25:1901-1907
Nakayama Y, Hayakawa K, Mitsuhashi N, Saito Y, Niibe H (1997) Long-term survivors of non-small cell lung cancer after radiation therapy: the significance of histological type. Anticancer Res 17:2769-2773

Nicholson RI, Gee JMW, Harper ME (2001) EGFR and cancer prognosis. Eur J Cancer 37(Suppl 4):S9-S15

Ohsaki Y, Tanno S, Fujita Y, Toyoshima E, Fujiuchi S, Nishigaki Y, Ishida S, Nagase A, Miyokawa N, Hirata S, Kikuchi K (2000) Epidermal growth factor receptor expression correlates with poor prognosis in non-small cell lung cancer patients with p53 overexpression. Oncol Rep 7:603-607

Organisation for Economic Co-operation and Development (2007) OECD series on principles of good laboratory practice and compliance monitoring (accessed 2 May 2007, through http:// www.oecd.org/document/63/0,2340,en_2649_34381_2346175_ 1_1_1_1,00.html)

Paez JG, Jänne PA, Lee JC, Tracy S, Greulich H, Gabriel S, Herman P, Kaye FJ, Lindeman N, Boggon TJ, Naoki K, Sasaki H, Fujii Y, Eck MJ, Sellers WR, Johnson BE, Meyerson M (2004) EGFR mutations in lung cancer: correlation with clinical response to gefitinib therapy. Science 304:1497-1500

Pocock SJ, Simon R (1975) Sequential treatment assignment with balancing for prognostic factors in the controlled clinical trial. Biometrics 31:103-115

Salomon DS, Brandt R, Ciardiello F, Normanno N (1995) Epidermal growth factor-related peptides and their receptors in human malignancies. Crit Rev Oncol Hematol 19:183-232

Shepherd FA, Rodrigues PJ, Ciuleanu T, Tan EH, Hirsh V, Thongprasert S, Campos D, Maoleekoonpiroj S, Smylie M, Martins R, van Kooten M, Dediu M, Findlay B, Tu D, Johnston D, Bezjak A, Clark G, Santabarbara P, Seymour L (2005) Erlotinib in previously treated non-small-cell lung cancer. N Engl J Med 353:123132

Slamon DJ, Godolphin W, Jones LA, Holt JA, Wong SG, Keith DE, Levin WJ, Stuart SG, Udove J, Ullrich A (1989) Studies of the HER-2/neu proto-oncogene in human breast and ovarian cancer. Science 244:707-712

Sordella R, Bell DW, Haber DA, Settleman J (2004) Gefitinib-sensitizing EGFR mutations in lung cancer activate anti-apoptotic pathways. Science 305:1163-1167

Spaulding DC, Spaulding BO (2002) Epidermal growth factor receptor expression and measurement in solid tumors. Semin Oncol 29:45-54

Thatcher N, Chang A, Parikh P, Rodrigues PJ, Ciuleanu T, von Pawel J, Thongprasert S, Tan EH, Pemberton K, Archer V, Carroll K (2005) Gefitinib plus best supportive care in previously treated patients with refractory advanced non-small-cell lung cancer: results from a randomised, placebo-controlled, multicentre study (Iressa Survival Evaluation in Lung Cancer). Lancet 366:15271537

Tsao M-S, Sakurada A, Cutz J-C, Zhu C-Q, Kamel-Reid S, Squire J, Lorimer I, Zhang T, Liu N, Daneshmand M, Marrano P, da Cunha Santos G, Lagarde A, Richardson F, Seymour L, Whitehead M, Ding K, Pater J, Shepherd FA (2005) Erlotinib in lung cancermolecular and clinical predictors of outcome. N Engl J Med 353:133-144

Veale D, Kerr N, Gibson GJ, Kelly PJ, Harris AL (1993) The relationship of quantitative epidermal growth factor receptor expression in non-small cell lung cancer to long term survival. $\mathrm{Br} \mathrm{J}$ Cancer 68:162-165

Volm M, Rittgen W, Drings P (1998) Prognostic value of ERBB-1, VEGF, cyclin A, FOS, JUN and MYC in patients with squamous cell lung carcinomas. Br J Cancer 77:663-669

Woodburn JR (1999) The epidermal growth factor receptor and its inhibition in cancer therapy. Pharmacol Ther 82:241-250 